\title{
Carnitine and Sepsis: A Review of an Old Clinical Dilemma
}

\author{
Shima Hatamkhani, Iman Karimzadeh, Sepideh Elyasi, Shadi Farsaei, Hossein Khalili \\ Department of Clinical Pharmacy, Faculty of Pharmacy, Tehran University of Medical Sciences, Tehran, Iran
}

Received March 28, 2013; Revised, May 7, 3013; Accepted May 15, 2013; Published, May 21, 2013.

\begin{abstract}
Purpose. The precise role of carnitine as the key regulator of lipid metabolism in sepsis is unclear. In this review, available experimental as well as clinical evidences regarding the probable beneficial effects of carnitine in sepsis were evaluated. Method. A comprehensive literature search was performed in the related medical databases. Related experimental and clinical studies were included. Results and Conclusion: The plasma and tissue level of carnitine or its derivatives in septic condition is variable and inconclusive. Survival and outcomes are considered in only few studies. Despite its favorable safety profile, due to limited clinical evidence, it seems reasonable not to currently consider carnitine as a mandatory and beneficial supplement under septic conditions. Further well-designed, standard clinical trials are warranted in this regards.
\end{abstract}

This article is open to POST-PUBLICATION REVIEW. Registered readers (see "For Readers") may comment by clicking on ABSTRACT on the issue's contents page.

\section{INTRODUCTION}

Carnitine is an amino acid derivative which is synthesized endogenously from the essential amino acids lysine and methionine (1). Although the initial steps of carnitine synthesis occur in most mammalian tissues, the main final reaction, hydroxylation of $\gamma$-butyrobetaine to carnitine, take place only in a few organs. It seems that the liver is the main site for this reaction in rats, while in humans, carnitine is synthesized in kidney, liver, and brain where the essential enzyme, $\gamma$ butyrobetaine hydroxylase is located (2). Carnitine is stored mainly in muscles.

Carnitine has an important role in facilitating medium- and long-chain fatty acid transport from the cytosol into mitochondria for $\beta$-oxidation and energy generation. In addition, carnitine stimulates pyruvate dehydrogenase complex activity and the Krebs cycle, increasing branched-chain amino acids oxidation in muscles (3).

Oral dosage forms of carnitine are currently approved by the US Food and Drug Administration for primary systemic and secondary carnitine deficiency. In addition, intravenous carnitine is approved in acute and chronic treatment of patients with secondary carnitine deficiency (4-6) as well as prevention and treatment of carnitine deficiency in patients with end-stage renal disease (ESRD) undergoing hemodialysis (7-15). Some unlabeled uses of carnitine are treatment or prophylaxis of valproate toxicity $(16,17)$, Alzheimer's disease (18), muscle disorders (19), cardiovascular diseases (20), HIV infection $(18,21)$, and male infertility (22).

In addition to de novo synthesis, carnitine is being obtained exogenously. Meat is known to be the best food source for exogenous carnitine. Milk, dairy products, seafood, and fish take the next place, while vegetables contain a negligible amount of carnitine (23). Large amounts of carnitine (20$200 \mathrm{mg} /$ day for a $70 \mathrm{~kg}$ individual) along with essential amino acids and micronutrients needed for endogenous carnitine biosynthesis could be provided by a regular balanced diet. Even in strict vegetarian diets (as little as $1 \mathrm{mg} /$ day exogenous carnitine for a $70 \mathrm{~kg}$ person), endogenous synthesis combined with the high renal tubular reabsorption rate is enough to prevent deficiency in generally healthy people. Hence, carnitine deficiency seems to be uncommon in the healthy, well-nourished adult people (24).

There are 2 distinct types of carnitine deficiency, including primary and secondary deficiency. Primary carnitine deficiency, a rare autosomal recessive disorder, is resulted from

Corresponding Author: Dr. Hossein Khalili, Department of Clinical Pharmacy, Faculty of pharmacy, Tehran University of Medical Sciences, EnghelabAve,Tehran, Iran; E-mail: khalilih@tums.ac.ir 
genetic defect in the membrane carnitine transporter in the muscle. In this disorder, plasma free carnitine values are below $5 \mu \mathrm{M}$, much less than its normal range defined as $25-50 \mu \mathrm{M}(25,26)$. Primary carnitine deficiency was first described as muscle carnitine deficiency in 1973 (27). Following this, systemic carnitine deficiency associated with concurrent hepatic encephalopathy and myopathy as well as progressive cardiomyopathy was also reported (28). Assuming the overlapped clinical manifestations of the hepatic, myopathic, and cardiac forms, most clinicians consider systemic carnitine deficiency as a single entity.

Secondary carnitine deficiency is related to some inborn errors of metabolism and acquired medical conditions such as organic aciduria, mitochondrial respiratory chain defects, methylene tetrahydrofolate reductase deficiency, and treatment with valproate (29), zidovudine, and pivampicillin (30-31). It also can be caused by impaired proximal renal tubule carnitine reabsorption and incomplete carnitine biosynthesis in immature neonates with $\gamma$ butyrobetaine hydroxylase deficiency (2). Chronic conditions including kidney diseases, diabetes mellitus, heart failure, cirrhosis, fanconi syndrome, malabsorption, and alzheimer's disease have been also associated with secondary carnitine deficiency. Besides chronic conditions, secondary carnitine deficiency has been also observed in critical settings such as trauma, sepsis, and acute organ failure, resulted in increased need for exogenous carnitine. Septic patients may also vulnerable to carnitine deficiency due to acquired liver and kidney dysfunction $(16,32)$. Carnitine deficiency was reported in hypermetabolic critically ill patients following parenteral nutritional support which may be related to the fact that available total parenteral nutrition (TPN) solutions are carnitine free (33). It is hypothesized that long term critically ill patients progressed to sepsis may be suffered from carnitine deficiency and its related complications such as immune system malfunction (32).

Sepsis defined as a severe and overwhelming inflammatory response to an infection that may progress to multiple organ dysfunction syndromes (MODS) with high morbidity and mortality (34-37). Blood flow redistribution and changes in substrate utilization in sepsis can lead to hypermetabolic state in association with tissue hypoperfusion and inadequate oxygenation $(38,39)$. In excessive stress, energy sources will be switched preferably from carbohydrates to more dense ones such as lipids. Thus, these circumstances allow carbohydrates to move toward those tissues like central nervous system (CNS) which has the obligatory pathway of glucose consumption (1). This metabolic adaption is evidenced by low respiratory quotient (RQ) even when there are sufficient carbohydrate sources (40). Sepsis and endotoxaemia result in impaired lipid metabolism and hepatic energy generation from fatty acid oxidation (41). In patients with sepsis, cells are more susceptible to inflammatory mediators such as TNF and lipopolysacharide (LPS) due to abnormal carnitine metabolism which may lead to septic shock syndrome and multiple organ failure (42).

Carnitine prevents accumulation of toxic acyl Coenzyme A (CoA) compounds and insufficient energy production which may occur during sepsis by regulating ratio of acyl-CoA to free $\mathrm{CoA}$ in the mitochondria $(16,43)$. A number of experimental and clinical studies have been demonstrated carnitine serum or plasma level abnormality in septic conditions (44-49).

Considering the fact that lipid metabolism impaired in sepsis, carnitine might exert its beneficial effects through enhancing lipid oxidation and energy formation. In this review, available published experimental as well as clinical data regarding the role of carnitine administration in sepsis were collected and discussed. To the best of our knowledge, this is first review that have gathered and evaluated all animal and human published data on carnitine supplementation in sepsis.

\section{METHODS}

A comprehensive literature search was performed using relevant key words including "carnitine", "Lcarnitine", "levo-carnitine", "acetyl-carnitine", "sepsis", "septic", "septic shock", "critically ill" and "severe sepsis" in the following databases: Scopus, Medline, Embase, Google scholar, Cochrane central register of controlled trials, and Cochrane database systematic reviews. Bibliographies of eligible articles were also examined for additional relevant studies. No time limitation was considered in this review. Thirty four articles regarding carnitine level measurement in septic condition were found. The search was limited by focusing on only interventional studies about carnitine administration in sepsis. Non-English language articles, congress abstracts, as well as in vitro studies were considered ineligible for inclusion. 


\section{Non-clinical studies}

Four studies have focused specifically on the effects of carnitine administration in experimental models of sepsis. In the first one published by Takeyama et al. in 1989, sepsis was induced by intraperitoneal administration of Escherichia coli lipopolysaccharide (LPS) in rats. L-carnitine (500 $\mathrm{mg} / \mathrm{kg}$ ) injected intraperitoneally at $16 \mathrm{~h}$ and $30 \mathrm{~min}$ before LPS administration. There were 2 control groups receiving saline instead of L-carnitine and saline instead of LPS, respectively. After LPS injection, rats were checked for survival every $1 \mathrm{~h}$ for $24 \mathrm{~h}$. The concentrations of CoA and carnitine derivatives (except for malonyl-CoA) declined in the liver following LPS administration while the level of hepatic malonyl-CoA increased significantly. This suggests that LPS causes activated fatty acids to undergo esterification and lipogenesis rather than oxidation. L-carnitine treatment before LPS injection increased survival rate significantly $(\mathrm{P}<0.05)$, but did not affect enzymes involved in metabolizing fatty acids, CoA, or carnitine derivatives in the liver. L-carnitine plus LPS administration was associated with higher plasma levels of free L-carnitine and total acid soluble carnitine at $6 \mathrm{~h}$ than L-carnitine plus saline. This might be due to alteration in the rate of exogenous L-carnitine absorption from abdominal cavity or its excretion from the circulation. Although exogenous L-carnitine had a considerable effect on hepatic levels of carnitine and its esters, but it did not alter the level of ketone bodies and CoA. Actually, they decreased exactly at the same manner in the group received saline plus LPS. Not only free hepatic carnitine, but also total acidsoluble carnitine as well as acid-insoluble acylcarnitine increased after L-carnitine administration. This could be due to the fact that exogenous Lcarnitine has access to acyl-CoA pool through the activity of carnitine acyltransferase (50).

In 1993 Gallo et al evaluated the potential role of carnitine and its derivative, 2-hydroxybutyryl ester of isovalerylcarnitine tartrate (VBT carnitine) in normalizing hepatic lipid metabolism in LPSinduced sepsis in rats (41). L-carnitine $(100 \mathrm{mg} / \mathrm{kg})$ or VBT carnitine (to deliver $100 \mathrm{mg} / \mathrm{kg}$ carnitine) was injected intraperitoneally at $-16 \mathrm{~h},-8 \mathrm{~h}$, and zero time in the carnitine-treated group. Within $2 \mathrm{~h}$ of LPS injection, all 14 and 17 rats in carnitine- and VBT carnitine-treated groups were within fair to normal health status, respectively compared to only 4 out of 11 rats in the control group. By 30 h, only 3 out of 9 survivors from the control group considered as fair to normal; whereas, all survived animal in carnitine- $(\mathrm{n}=9)$ and VBT carnitinetreated groups $(n=12)$ were within fair to normal health status. Finally over $96 \mathrm{~h}$ observation period, carnitine and VBT carnitine may have declined the lethality of LPS-induced sepsis since there were 7 and 5 deaths in these groups, respectively compared to 8 deaths in the control group. So, administration of both carnitine and VBT carnitine markedly delayed and decreased the severity of sepsis and hastened recovery. Along with less illness in carnitine or VBT carnitine-treated groups, greater food consumption and less weight loss were also observed. In addition, serum triglyceride (TG) was significantly lower in both carnitine treatment compared to control groups. This might be related to decrease in hepatic synthesis of fatty acids and decline in fatty acid moving toward esterification. Moreover, liver lipogenesis in the control group was significantly increased compared to both carnitine-treated groups. In this study, liver carnitine level increased in both control and carnitine-treated groups. Although increase in liver carnitine level is an unexpected finding in sepsis, but it may be regulated by glucagon which increases in sepsis. The results of this study demonstrated the striking effects of carnitine and VBT carnitine in the health of septic rats. There were no detectable differences in effectiveness between carnitine and its derivative, but VBT carnitine seems to be more effective in the treatment of heart muscle disease and brain ischemia. According to results of this study, the authors suggested that carnitine may have a therapeutic role in sepsis treatment through changing fatty acid metabolism from the esterification to oxidation pathway (41).

In 1996, Yamakawa et al (51) assessed the distribution of carnitine in rats with sepsis or acute liver failure. Sepsis was induced by cecal ligation and puncture. Five rats each in the control, sepsis and acute liver failure groups were given a single intravenous injection of carnitine $(250 \mathrm{mg} / \mathrm{kg})$ via the tail vein. They were killed $2 \mathrm{~h}$ later. In the sepsis group before carnitine administration, the muscle free carnitine level was significantly decreased compared to that in the control group. The hepatic free carnitine level also decreased in the sepsis group. Short-chain acyl-carnitine was increased in adipose tissue, while both free carnitine and short-chain acyl-carnitine were increased in the epididymis compared with levels in the control group. No significant differences were noted in 
other tissues. After L-carnitine administration, the concentrations of free carnitine and short-chain acyl-carnitine increased in the liver and kidneys, while the renal level of long-chain acyl-carnitine decreased. These findings demonstrated the accumulation of exogenous free carnitine as well as the transport and synthesis of endogenous free carnitine and short-chain acyl-carnitine. Most shortchain acyl-carnitine may be acetylcarnitine, which is probably produced by the transfer of acetyl groups or fatty acid oxidation. Muscle levels of free carnitine and short-chain acyl-carnitine also increased in a less marked degree; although free carnitine level was nearly restored to the control value, probably because that carnitine pool in muscle was too large to be influenced by the dose of exogenous carnitine. According to these findings, it can be concluded that exogenous free carnitine accumulated directly in organs of septic rats involved with carnitine deficiency, suggesting a role for carnitine in the regulation of lipid metabolism in sepsis (51).

Inflammatory cytokines have been demonstrated to play a pivotal role in altering overall metabolism in several experimental and clinical models of diseases-induced cachexia such as sepsis, cancer, and AIDS. Successful management of cachexia could reduce the discomfort and enhance the quality life of affected patients. Several modalities of treatment have been studied for achieving this purpose. In the last study from experimental series published in 1995, Winter et al. investigated the potential effect of L-carnitine on the level of plasma TG as well as inflammatory cytokines including TNF- $\alpha$, IL- $1 \beta$, and IL- 6 in 2 types of rat models, cachexia due to sarcoma and septic shock. In septic shock model, sepsis was induced by the intraperitoneal injection of Escherichia coli LPS. At -16, -8, 0, and $8 \mathrm{~h}$ post LPS injection, rats received either carnitine (100 $\mathrm{mg} / \mathrm{kg}$ ) in buffer or buffer only intraperitoneally. In sarcoma-induced cachexia model, sarcoma was developed by subcutaneous implanting of methylcholanthrene on the left flank of rats. They were divided into 2 subgroups receiving either carnitine continuously $(200 \mathrm{mg} / \mathrm{kg} /$ day $)$ via implanted osmotic pumps or buffer for $7,14,21$, or 28 days. In LPS-induced sepsis model, carnitine significantly lowered the level of illness $(\mathrm{P}<0.001)$ and improved food consumption. In addition, carnitine-treated compared to control rats demonstrated a significantly lower plasma level of TG $(45 \pm 6$ vs. $83 \pm 8 \mathrm{mg} / \mathrm{dl}$, respectively; $\mathrm{P}<$
0.001). The serum level of TNF- $\alpha$ in carnitinetreated group were significantly lower than that of the control group $(\mathrm{P}<0.02)$. In sarcoma-induced cachexia model, food consumption, rat body, and tumor weight did not differ significantly between control and carnitine-treated rats. In contrast, carnitine treatment for 7 days significantly decreased the tumor-induced rise in TG level ( $\mathrm{P}<$ 0.04). Furthermore, plasma concentrations of TNF$\alpha$ and IL-1 $\beta$ were significantly lower following 28 days of receiving L-carnitine compared to control rats $(\mathrm{P}<0.04)$. Regarding the profile of plasma carnitine level, it reached the highest level 7 days after pump implantation but decreased to almost control level over the next 21 days. The authors suggested that obstruction of pumps by host tissue growth could decrease carnitine delivery. This might account for the failure of carnitine treatment in lowering TG levels for longer than 7 days in sarcoma-induced cachexia model. The results of this study demonstrated that carnitine treatment has beneficial effects on morbidity as well as lipid metabolism in 2 experimental models of LPSinduced septic shock and sarcoma-induced cachexia partially through reducing serum levels of inflammatory cytokines (52).

The aforementioned experimental investigations examined the role of carnitine in different septic animal models. Septic state was induced by cecal ligation and puncture, LPS or live bacteria injection. This may partially account for some inconclusive results of these studies. As plasma carnitine might not show an accurate estimation of total body carnitine pool, animal models allow measurement of tissue level of carnitine which may be difficult to be done in human subjects. Few studies have considered survival as their endpoint. Carnitine injection increased survival rate significantly without affecting its level in liver which may be justified by altered carnitine absorption from peritoneum or its elimination from the blood (5). Carnitine could improve sepsis severity in septic rats $(41,46)$. It has favorable role in normalizing lipid metabolism in sepsis. The results of carnitine values are variable and controversial depending on measuring carnitine level as free carnitine, acylcarnitine, or total carnitine in blood, urine, muscles, myocardium, liver, or other tissues. Similarly, this issue has been noticed by Eaton et al in their review on carnitine and fatty acid oxidation in sepsis (1). Regarding the above drawbacks, results of the available animal studies should be validated and could be only 
Table 1. Carnitine supplementation in sepsis, animal data

\begin{tabular}{|c|c|c|c|c|}
\hline $\begin{array}{l}\text { Study } \\
\text { (Reference) }\end{array}$ & Subjects (Number) & Intervention group & $\begin{array}{l}\text { Evaluated } \\
\text { parameters }\end{array}$ & Main result(s) \\
\hline $\begin{array}{l}\text { Winter et al, } \\
1995(52)\end{array}$ & $\begin{array}{l}\text { Rats with LPS- } \\
\text { induced sepsis (not } \\
\text { determined) or } \\
\text { sarcoma-induced } \\
\text { cachexia (35) }\end{array}$ & $\begin{array}{l}\text { LPS-induced sepsis: } \\
\text { L-carnitine ( } 100 \mathrm{mg} / \mathrm{kg}) \\
\text { intraperitoneally at }-16,-8 \text {, } \\
0 \text {, and } 8 \text { h post LPS } \\
\text { Sarcoma-induced } \\
\text { cachexia: L-carnitine } \\
\text { continuously ( } 200 \\
\text { mg/kg/day) via } \\
\text { subcutaneous implanted }\end{array}$ & $\begin{array}{l}\text { Serum level of } \\
\text { triglyceride, TNF- } \\
\alpha, \text { IL-1 } \beta \text {, and IL-6, } \\
\text { L-carnitine; } \\
\text { Health status, } \\
\text { body weight, food } \\
\text { consumption, } \\
\text { tumor volume. }\end{array}$ & $\begin{array}{l}\text { L-carnitine treatment has } \\
\text { beneficial effects on morbidity } \\
\text { as well as lipid metabolism in } \\
\text { experimental models of LPS- } \\
\text { induced septic shock and } \\
\text { sarcoma-induced cachexia } \\
\text { partially through reducing } \\
\text { serum levels of inflammatory } \\
\text { cytokines. }\end{array}$ \\
\hline
\end{tabular}

Takeyama et Rats with LPS- L-carnitine $(500 \mathrm{mg} / \mathrm{kg})$ al, 1989 (50) induced sepsis

Yamakawa et al, 1996 (51)

Rats with sepsis or acute liver failure (10)

Gallo et al, Lipopolysaccharide1993 (41) induced sepsis in rats (31)
A single intravenous injection of L-carnitine $(250 \mathrm{mg} / \mathrm{kg})$ via the tail vein

L-carnitine $(100 \mathrm{mg} / \mathrm{kg})$ or VBT carnitine (to deliver $100 \mathrm{mg}$ carnitine $/ \mathrm{kg}$ ) was injected intraperitoneally, at $-16 \mathrm{~h},-8 \mathrm{~h}$, and at zero time in the carnitinetreated groups. The final dose of carnitine was administered at $8 \mathrm{hr}$ postLPS in the carnitinetreated groups
Total and free carnitine concentration, CoASH, acetyl$\mathrm{CoA}$, and malonyl-CoA levels.

Free L-carnitine as well as acylcarnitine concentrations in the heart, gastrocnemius, liver, kidneys, adipose tissue and epididymis

Health status, serum carnitine an triglycerides level, food consumption and lipogenesis

\section{L-carnitine treatment before LPS injection increased survival rate significantly but did not affect enzymes involved in metabolizing fatty acids, CoA, or carnitine derivatives in the liver.}

The concentrations of free Lcarnitine and short-chain acylcarnitine increased in the liver and kidneys, while the renal level of long-chain acylcarnitine decreased after Lcarnitine administration.

Carnitine and its derivative, isovalerylcarnitine tartrate markedly delayed and decreased the severity of illness, hastened recovery, and serum triglycerides in sepsis

Carnitine may have a therapeutic role in sepsis treatment partially through changing fatty acid metabolism from the esterification to oxidation pathway considered as a guide for performing clinical trials in this area. Details of non-clinical studies on the effect of carnitine administration on sepsis are given in Table 1.

\section{Clinical studies}

For the first time, beneficial effects of carnitine treatment in 2 critically ill patients were reported by Carlsson et al. in 1984 (40). In that study, carnitine was infused to 2 mechanically ventilated male patients with peritonitis and septicemia who were receiving TPN. The blood pressure in one patient was maintained by dopamine infusion and both were receiving insulin along with their TPN (50\% fat and 50\% glucose) to control their blood glucose levels. Carnitine administration (2 g) reduced insulin requirement by $70 \%$ after $24 \mathrm{~h}$ in one patient 
and resulted in withdrawal of dopamine after $48 \mathrm{~h}$ of carnitine infusion $(6 \mathrm{~g})$ in another patient. Nitrogen balance was also improved (40). Although favorable effects of carnitine therapy in this study were demonstrated only in 2 patients, it opened a new window for research on carnitine therapy in critically ill patients with sepsis. Consequently, another study was performed in 1985 regarding acyl-carnitine administration $(100 \mathrm{mg} / \mathrm{kg} / \mathrm{day})$ in 19 patients under TPN (33). In this study, acylcarnitine was administered in 8 non-septic surgical patients and 11 septic patients; whereas 20 surgical patients without any evidence of infection or malnutrition were considered as the control group. During acyl-carnitine infusion, plasma TG significantly reduced in non-septic compared to the control group. Although the decrease of $37 \%$ and $23 \%$ in hydroxybutyrate levels were also detected in non-septic and septic patients following acylcarnitine infusion, respectively, these differences were not statistically significant compared to the control group. Moreover, it is interesting to notice that during acyl-carnitine infusion, RQ decreased significantly only in septic patients which may be related to decrease in $\mathrm{CO}_{2}$ production. This showed the important role of carnitine in the oxidative metabolism in septic patients with hypermetabolic state. Reduction of RQ in septic patients may be associated with a utilization of substrates characterized by low RQ such as branched-chain amino acids and fatty acids rather than glucose and decline in directing glucose carbon to the lipogenesis pathway (33).

Various aspects of immune system functions such as mitogen responsiveness of lymphocytes become compromised in traumatic injury patients. On the other hand, the ability of immune system following trauma is essential to combat against sepsis (53). Lymphocytes mainly utilize glucose (54), but most mitochondria of lymphoblasts are capable of oxidizing long-chain fatty acid which is carnitine-dependent (55). Carlsson et al. in 1987 evaluated the stimulatory effect of intravenous (IV) carnitine supplementation (total dose of $110 \mathrm{mg} / \mathrm{kg}$ during a period of 4 days) on in vitro mitogenesis of lymphocytes obtained from 3 traumatic patients with severe post-operative infections (32). Augmenting culture of lymphocytes with carnitine markedly improved thymidine uptake. So, suppression of mitogenic response can be restored by the addition of carnitine to the culture medium. The rate of spontaneous thymidine uptake in patients received carnitine was comparable to that observed in healthy control patients. However in one patient, the high spontaneous thymidine uptake and the poor mitogenic response of lymphocytes, turned toward normal after carnitine supplementation. This normalized response was less influenced by the presence of carnitine in the culture medium of lymphocytes because adding carnitine to the culture medium actually worsened the poor mitogenic response of lymphocytes. Three patients received IV carnitine improved clinically and resumed spontaneous breathing, however, patients who did not receive carnitine supplementation were ventilator-dependent. Although carnitine improved mitogenic response in traumatic patients with infection, but no effect of carnitine on in vitro lymphocyte activation was identified in healthy control subjects. It is unclear if these different changes in thymidine utilization can be associated with carnitine supplementation or the medical and nutritional status of the patients. The probable beneficial effect of carnitine supplementation in traumatic septic patients may be restoration of lymphocytic carnitine deficiency.

As discussed before, supplementation of carnitine is decreased in septic patients under TPN (56), but also the urinary elimination of carnitine is increased in these patients. Therefore it seems that additional supplementation of carnitine is required to increase the catabolism of infused lipid in septic patients under TPN $(33,57)$. Accordingly, Gibault et al. conducted a study in 1988 to evaluate effects of carnitine supplementation on catabolism of infused lipids in septic patients (58). Along with starting daily infusion of Intralipid 20\%, 2 g/day carnitine was infused intravenously. As expected, free, total, and ratio of free/total carnitine levels rose in the intervention group during Intralipid infusion but, these parameters were comparable to healthy subjects' values and were within the normal limit. Although the ratio of free/total carnitine increased significantly until $4 \mathrm{~h}$, but at $6 \mathrm{~h}$, they returned to basal level. No significant difference was seen between 2 groups regarding TG level $4 \mathrm{~h}$ after the complementation of Intralipid infusion. However, lipid plasma level decreased rapidly when Intralipid infusion was stopped. Interestingly, this decline was more quickly in the presence of carnitine. It seems that carnitine increased the uptake and hepatic oxidation of circulating fatty acids after complementation of Intralipid infusion. The mean acetoacetate and $\beta$-hydroxybutyrate levels increased higher and faster in carnitinetreated than control groups between 0 and $4 \mathrm{~h}$, but 
the average concentrations of ketone bodies were insignificantly lower in carnitine group at 6 and $8 \mathrm{~h}$. It means that exogenous fatty acids were converted more rapidly into ketone bodies in the presence of carnitine. Decrease in the non-esterified fatty acids/ $\beta$-hydroxybutyrate ratio observed during carnitine supplementation might be considered as the marker of reestablishment of hepatic mitochondrial ketogenic capacity of infused fatty acids. The concomitant administration of carnitine with Intralipid attenuated the lactate and pyruvate plasma levels following Intralipid infusion. Different mechanisms have been proposed by the authors to justify carnitine effects in decreasing pyruvate level such as increasing the inflow of pyruvate in the Krebs cycle, reducing the rate of anaerobic glycolysis, and enhancing the elimination of acetyl radicals from the mitochondria (59). Finally, it seems that carnitine improved the uptake rate and oxidation of exogenous fatty acids by liver. Details of clinical studies regarding carnitine administration in sepsis were shown in table 2.

\section{CONCLUSION}

Carnitine is considered as a pivotal mediator of fatty acid oxidation that is the human preferred metabolic pathway during sepsis and critical conditions. To the best of our knowledge, this is first review that have gathered and evaluated all animal and human published data on carnitine supplementation in sepsis.

Table 2. Carnitine supplementation in sepsis, human data

Reference Study design $\quad$ Subjects (Number)

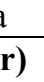

Mechanically ventilated

male patients with

peritonitis and septicemia

received TPN (2)

\begin{tabular}{lll}
\hline Carlsson et & Case report & Mechanically ventilated \\
al, $1984(40)$ & & male patients with \\
& peritonitis and septicemia \\
& received TPN (2)
\end{tabular}

Nanni et al, Controlled trial 1985 (33)

Carlsson et Not determined al, 1987 (32)

Gibault et al, Randomized, 1988 (58) controlled trial ;non-septic surgical patients and 11 septic patients) received acylcarnitine (19)

Surgical patients without evidence of infection or malnutrition were considered as control group (20)

Traumatic patients with severe post-operative infections (3)

Healthy control subjects

Septic patients received carnitine (13)

Septic patients without carnitine (control) (15)
Patients during TPN (8

Intervention Main result(s)

The total
cumulative doses
of 2 and $6 \mathrm{~g}$ of
carnitine were
infused during 24
$\mathrm{hr}$ and $48 \mathrm{hr}$,
respectively

Acyl-carnitine infused (100 $\mathrm{mg} / \mathrm{kg} /$ day) consistent with TPN administration

IV carnitine supplementation (total dose of 110 $\mathrm{mg} / \mathrm{kg}$ during a period of 4 days )

Carnitine (2 g/day) was infused IV along with starting daily infusion of Intralipid
Reduction in insulin requirement in one and withdrawal of dopamine in another patient. Nitrogen balance improvement

Acyl-carnitine infusion decreased significantly the respiratory quotient in septic patients suggesting its important role in the oxidative metabolism in hypermetabolic state.

Patients received IV carnitine improved clinically and resumed spontaneous breathing, however, patients not received carnitine supplementation required mechanical ventilation.

Carnitine increases the uptake and hepatic oxidation of circulating fatty acids after complementation of Intralipid infusion.

Exogenous fatty acids are converted more rapidly into ketone bodies in the presence of carnitine. 
The results of clinical and non-clinical studies are inconclusive depending on measuring carnitine level as free carnitine, acylcarnitine, or total carnitine in blood, urine, muscles, myocardium, liver, or other tissues. As plasma carnitine did not show an accurate estimation of total body carnitine pool, animal models allow measurement of tissue level of carnitine which may be difficult to be done in human subjects. Few studies have considered survival as their endpoint.

Carnitine is generally considered to be a safe supplement at least in healthy adults. Unpleasant fishy body or urine odor (probably due to the formation of trimethylamine), flatulence, nausea, and vomiting, headache, pruritus and rash are common adverse reactions of high doses (up to $6000 \mathrm{mg}$ per day) of carnitine in various clinical settings (60). No clinically significant adverse reaction was reported in the available studies. However its safety profile in long term supplementation is remained to be determined.

There are limited evidences regarding carnitine administration in septic conditions especially in clinical setting. On the other hand, relevant clinical studies are not well-designed, their sample sizes are relatively small, and few of them have considered survival and outcome as their endpoint. Limited data on carnitine levels in septic patients prevents having consistent evaluation of carnitine deficiency in this population which necessitates further investigation on this issue. Notably, all of subjects in human studies received carnitine parentally and no study has evaluated the efficacy of other administration routes of carnitine (such as orally or via enteral feeding tube). Due to these limitations, it is difficult to achieve a particular consensus about the precise role of carnitine supplementation in sepsis. However based on the favorable safety profile along with the documented effects of Lcarnitine in lipid metabolism as well as the pivotal role of lipid metabolism in providing adequate energy in sepsis, present data are encouraging but these need to be confirmed with further well designed standard clinical trials with large sample size.

\section{Future perspective}

There are limited experimental and clinical evidences regarding carnitine administration in septic conditions. On the other hand, relevant clinical studies are not well-designed, their sample sizes are relatively small, and few of them have considered survival and outcome as their endpoint.
Controversial data on carnitine levels in septic patients disables having consistent evaluation of carnitine deficiency in this population which necessitates further investigations on this issue. Notably, all subjects in human trials received carnitine via parenteral route and no study has evaluated the efficacy of other administration routes of carnitine such as orally or via enteral feeding tube in sepsis. Regarding probable side effects, carnitine is generally considered to be a safe supplement at least in healthy adults. Unpleasant fishy body or urine odor (probably due to the formation of trimethylamine), flatulence, nausea, and vomiting, headache, pruritus, and rash are common adverse reactions of high doses (up to $6000 \mathrm{mg}$ per day) of carnitine in various clinical settings (54).

Due to these limitations, it is difficult to achieve a particular consensus about the precise role of carnitine supplementation in sepsis. However based on the favorable safety profile along with the documented effects of carnitine in lipid metabolism as well as the pivotal role of lipid metabolism in providing adequate energy in sepsis, performing further well-designed, standard clinical trials with large sample size are warranted in this regards.

\section{ACKNOWLEDGEMENT}

The authors of the manuscript do not have any competing interest about this work neither they received any funding for this work.

\section{REFERENCES}

1. Eaton S, Pierro A. Carnitine and fatty acid oxidation in sepsis. Monatshefte für Chemie/Chemical Monthly, 2005;136(8):1483-1492.

2. Rigault C, Le Borgne F, Demarquoy J. Genomic structure, alternative maturation and tissue expression of the human bbox1 gene. Biochim Biophys Acta, 2006;1761(12):1469-1481.

3. Xi L, Brown K, Woodworth J, Shim K, Johnson B, Odle J. Maternal dietary l-carnitine supplementation influences fetal carnitine status and stimulates carnitine palmitoyltransferase and pyruvate dehydrogenase complex activities in swine. J Nutr, 2008;138(12):2356-2362.

4. Angelini C. Carnitine deficiency. Lancet, 1975;2(7934):554.

5. Cederbaum SD, Auestad N, Bernar J. Four-year treatment of systemic carnitine deficiency. N Engl J Med, 1984;310(21):1395-1396.

6. Chapoy PR, Angelini C, Brown WJ, Stiff JE, Shug AL, Cederbaum SD. Systemic carnitine deficiency-- 
a treatable inherited lipid-storage disease presenting as reye's syndrome. N Engl J Med, 1980;303(24):1389-1394.

7. Vesela E, Racek J, Trefil L, Jankovy'ch V, Pojer M. Effect of 1-carnitine supplementation in hemodialysis patients. Nephron, 2001;88(3):218223.

8. Weschler A, Aviram M, Levin M, Better OS, Brook JG. High dose of 1-carnitine increases platelet aggregation and plasma triglyceride levels in uremic patients on hemodialysis. Nephron, 1984;38(2):120124.

9. Bohmer T. Conversion of butyrobetaine to carnitine in the rat in vivo. Biochim Biophys Acta, 1974;343(3):551-557.

10. Sakurauchi Y, Matsumoto Y, Shinzato T, Takai I, Nakamura Y, Sato M, et al. Effects of 1-carnitine supplementation on muscular symptoms in hemodialyzed patients. Am J Kidney Dis, 1984;32(2):258-264.

11. Matsumoto Y, Sato M, Ohashi H, Araki H, Tadokoro M, Osumi Y, et al. Effects of 1-carnitine supplementation on cardiac morbidity in hemodialyzed patients. Am J Nephrol, 2000;20(3):201-207.

12. Sloan RS, Kastan B, Rice SI, Sallee CW, Yuenger NJ, Smith B, et al. Quality of life during and between hemodialysis treatments: Role of 1-carnitine supplementation. Am J Kidney Dis, 1998;32(2):265272.

13. Hurot JM, Cucherat M, Haugh M, Fouque D. Effects of 1-carnitine supplementation in maintenance hemodialysis patients: A systematic review. J Am Soc Nephrol, 2002;13(3):708-714.

14. Eknoyan G, Latos DL, Lindberg J. Practice recommendations for the use of 1-carnitine in dialysis-related carnitine disorder. National kidney foundation carnitine consensus conference. Am J Kidney Dis, 2003;41(4):868-876.

15. Schreiber B. Levocarnitine and dialysis. Nutrition in clinical practice, 2005;20(2):218-243.

16. Lheureux PE, Penaloza A, Zahir S, Gris M. Carnitine in the treatment of valproic acid-induced toxicity - what is the evidence? Crit Care, 2005;9(5):431-440.

17. Warner MH, Anderson GD, Mccarty JP, Farwell JR. Effect of carnitine on measures of energy levels, mood, cognition, and sleep in adolescents with epilepsy treated with valproate. J Epilepsy, 1997;10(3):126-130.

18. Malaguarnera M. Carnitine derivatives: Clinical usefulness. Curr Opin Gastroenterol, 2012;28(2):166-176.

19. Jerusalem $F$, Engel AG, Sengupta $C$, Imbach $P$, Lammli J. Carnitine deficiency myopathy (author's transl)]. Dtsch Med Wochenschr, 1980;105(14):469473.

20. Ferrari R, Merli E, Cicchitelli G, Mele D, Fucili A, Ceconi C. Therapeutic effects of 1-carnitine and propionyl-1-carnitine on cardiovascular diseases: A review. Ann N Y Acad Sci, 2006;1033(1):79-91.

21. Rezaee H, Khalili H, Salamzadeh J, Jafari S, Abdollahi A. Potential benefits of carnitine in hivpositive patients. Future Virol, 2012;7(1):73-83.

22. Zhou X, Liu F, Zhai S. Effect of 1-carnitine and/or 1acetyl-carnitine in nutrition treatment for male infertility: A systematic review. Asia Pac J Clin Nutr, 2007;16 Suppl 1:383-390.

23. Demarquoy J, Georges B, Rigault C, Royer MC, Clairet A, Soty M, et al. Radioisotopic determination of 1-carnitine content in foods commonly eaten in western countries. Food Chem, 2004;86(1):137-142.

24. Lheureux PE, Hantson P. Carnitine in the treatment of valproic acid-induced toxicity.Clin Toxicol (Phila), 2009;47(2):101-111.

25. Magoulas PL, El-Hattab AW. Systemic primary carnitine deficiency: An overview of clinical manifestations, diagnosis, and management. Orphanet J Rare Dis, 2012;7(1):68.

26. Wasserstein AG. L-carnitine supplementation in dialysis: Treatment in quest of disease. Semin Dial, 2013;26(1):11-15.

27. Engel AG, Angelini C. Carnitine deficiency of human skeletal muscle with associated lipid storage myopathy: A new syndrome. Science, 1973;179(76):899-902.

28. Tripp ME, Katcher ML, Peters HA, Gilbert EF, Arya S, Hodach RJ, et al. Systemic carnitine deficiency presenting as familial endocardial fibroelastosis: A treatable cardiomyopathy. $\mathrm{N}$ Engl J Med, 1981;305(7):385-390.

29. Ito M, Ikeda Y, Arnez JG, Finocchiaro G, Tanaka K. The enzymatic basis for the metabolism and inhibitory effects of valproic acid: Dehydrogenation of valproyl-coa by 2-methyl-branched-chain acylcoa dehydrogenase. Biochim Biophys Acta (BBA)General Subjects, 1990;1034(2):213-218.

30. Holme E, Greter J, Jacobson CE, Lindstedt S, Nordin I, Kristiansson B, et al. Carnitine deficiency induced by pivampicillin and pivmecillinam therapy. Lancet, 1989;2(8661):469-473.

31. Proulx F, Lacroix J, Qureshi IA, Nadeau D, Gauthier M, Lambert M. Acquired carnitine abnormalities in critically ill children. Eur J Pediatr, 1997;156(11):864-869.

32. Carlsson M, Montan P, Sundqvist K. L-carnitine enhances lymphocyte mitogenesis in depleted traumatised and infected patients. Clin Nutr, 1987;6(1):39-44.

33. Nanni G, Pittiruti M, Giovannini I, Boldrini G, Ronconi P, Castagneto M. Plasma carnitine levels and urinary carnitine excretion during sepsis. JPEN J Parenter Enteral Nutr, 1985;9(4):483-490.

34. Proulx F, Gauthier M, Nadeau D, Lacroix J, Farrell CA. Timing and predictors of death in pediatric patients with multiple organ system failure. Crit Care Med, 1994;22(6):1025. 
35. Proulx F, Fayon M, Farrell CA, Lacroix J, Gauthier M. Epidemiology of sepsis and multiple organ dysfunction syndrome in children. Chest, 1996;109(4):1033-1037.

36. Wilkinson JD, Pollack MM, Glass NL, Kanter RK, Katz RW. Mortality associated with multiple organ system failure and sepsis in pediatric intensive care unit. J Pediatr, 1987;111(3):324-328.

37. Bone RC, Balk RA, Cerra FB, Dellinger RP, Fein AM, Knaus WA, et al. Definitions for sepsis and organ failure and guidelines for the use of innovative therapies in sepsis. The accp/sccm consensus conference committee. American college of chest physicians/society of critical care medicine. Chest, 2009;136(5 Suppl):e28.

38. Proulx F, Lacroix J, Farrell CA, Lambert M. Blood lactate and gastric intramucosal ph during severe sepsis. Crit Care Med, 1996;24(6):1092.

39. Dugas MA, Proulx F, De Jaeger A, Lacroix J, Lambert M. Markers of tissue hypoperfusion in pediatric septic shock. Intensive Care Med, 2000;26(1):75-83.

40. Carlsson M, Forsberg E, Thorne A. Observations during 1-carnitine infusion in two long-term critically ill patients. Clin Physiol, 1984;4(4):363365.

41. Gallo LL, Tian Y, Orfalian Z, Fiskum G. Amelioration of popolysaccharide-induced sepsis in rats by free and esterified carnitine. Mediators Inflamm, 1993;2(7):S51-56.

42. G. Famularo CDS, E. Arrigoni Martelli, and E. Jirillo. Carnitine and septic shock: A review. J Endotoxin Res, 1995;2(2):141-147.

43. Roe CR, Millington DS, Kahler SG, Kodo N, Norwood DL. Carnitine homeostasis in the organic acidurias. Prog Clin Biol Res, 1990;321:383-402.

44. Linz DN, Garcia VF, Arya G, Hug G, Tombragel E, Landrigan $\mathrm{E}$, et al. Weanling and adult rats differ in fatty acid and carnitine metabolism during sepsis. J Pediatr Surg, 1995;30(7):959-965; discussion 966.

45. Gullichsen E, Heinonen OJ, Nelimarkka O, Kuttila $\mathrm{K}$, Niinikoski J. Renal carnitine concentration decreases in endotoxic dogs. Critical Care Medicine, 1991;19(6):792.

46. Davis AT, Crady SK, Strong SA, Albrecht RM, Scholten DJ. Increased acylcarnitine clearance and excretion in septic rats. Biomed Biochim Acta, 1991; 50(1):81-86.

47. Lanza-Jacoby S, Holahan M, Reibel DK. Changes in tissue levels of carnitine during E. coli sepsis in the rat. Circ Shock, 1988;24(1):29-34.
48. Lanza-Jacoby S, Feagins K, Bodine R, Reibel DK. Impaired carnitine transport in the rat heart during $\mathrm{E}$. coli sepsis. Circ Shock, 1991;35(3):159-163.

49. Keskin S, Seven A, Mert M, Akalp F, Yurdakul F, Candan G. Could L-carnitine be an acute energy inducer in catabolic conditions? Dev Med Child Neurol, 1997:39(3):174-177.

50. Takeyama N, Takagi D, Matsuo N, Kitazawa Y, Tanaka T. Altered hepatic fatty acid metabolism in endotoxicosis: Effect of 1-carnitine on survival. Am J Physiol, 1989;256(1 Pt 1): E31-38.

51. Yamakawa M, Maeda J, Nakamura T, Mimura Y, Sugisaki K, Fujita $\mathrm{T}$, et al. Distribution of endogenous and exogenous carnitine in rats with sepsis and acute liver failure. Clin Nutr, 1996;15(3):133-140.

52. Winter BK, Fiskum G, Gallo LL. Effects of 1carnitine on serum triglyceride and cytokine levels in rat models of cachexia and septic shock. Br J Cancer, 1995;72(5):1173-1179.

53. Levy EM, Alharbi SA, Grindlinger G, Black PH. Changes in mitogen responsiveness lymphocyte subsets after traumatic injury: Relation to development of sepsis. Clin Immunol Immunopathol, 1984;32(2):224-233.

54. Hume DA, Hansen K, Weidemann MJ, Ferber E. Cytochalasin b inhibits lymphocyte transformation through its effects on glucose transport. Nature, 1987;272(5651):359.

55. Biberfeld P. Uropod formation in phytohaemagglutinin (pha) stimulated lymphocytes. Exp Cell Res, 1971;66(2):433-445.

56. Iapichino G, Radrizzani D, Colombo A, Ronzoni G. Carnitine excretion: A catabolic index of injury. JPEN J Parenter Enteral Nutr, 1988;12(1):35-36.

57. Fritz IB. Carnitine and its role in fatty acid metabolism. Adv Lipid Res, 1963;1:285-334.

58. Gibault JP, Frey A, Guiraud M, Schirardin H, Bouletreau P, Bach AC. Effects of 1-carnitine infusion on intralipid clearance and utilization. Study carried out in septic patients of an intensive care unit. JPEN J Parenter Enteral Nutr, 1988;12(1):29-34.

59. Opie LH. Role of carnitine in fatty acid metabolism of normal and ischemic myocardium. Am Heart J, 1979;97(3):375-388.

60. Hathcock JN, Shao A. Risk assessment for carnitine. Regul Toxicol Pharmacol, 2006;46(1):23-28. 